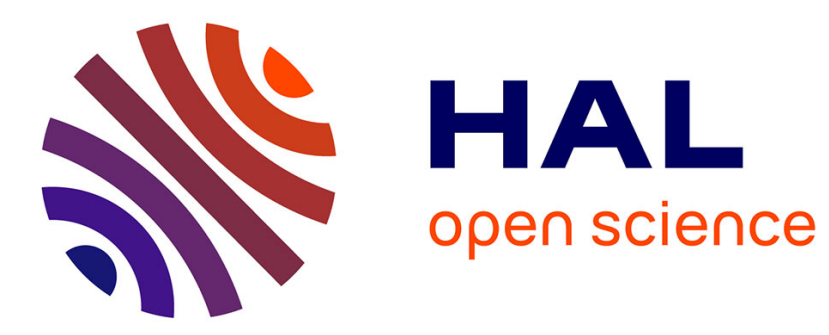

\title{
Nouvelles approches diachroniques et synchroniques dans le champ d'étude de la déviance et de la criminalité
} Frédéric Ocqueteau

\section{To cite this version:}

Frédéric Ocqueteau. Nouvelles approches diachroniques et synchroniques dans le champ d'étude de la déviance et de la criminalité. Déviance et Société, 1986, 10 (1), pp.1-19. hal-00932605

\section{HAL Id: hal-00932605 \\ https://hal.science/hal-00932605}

Submitted on 17 Jan 2014

HAL is a multi-disciplinary open access archive for the deposit and dissemination of scientific research documents, whether they are published or not. The documents may come from teaching and research institutions in France or abroad, or from public or private research centers.
L'archive ouverte pluridisciplinaire HAL, est destinée au dépôt et à la diffusion de documents scientifiques de niveau recherche, publiés ou non, émanant des établissements d'enseignement et de recherche français ou étrangers, des laboratoires publics ou privés. 


\title{
NOUVELLES APPROCHES DIACHRONIQUES ET SYNCHRONIQUES DANS LE CHAMP D'ÉTUDE DE LA DÉVIANCE ET DE LA CRIMINALITÉ
}

\author{
Fr. OCQUETEAU *
}

Cet article a pour but de faire état, à un moment où le besoin d'élargir le champ d'étude sur les appréhensions différentielles de la déviance et de la criminalité se fait sentir de manière récurrente dans la communauté scientifique, d'une réflexion personnelle qui tente d'en faire le point.

Il s'agirait moins de s'interroger sur les présupposés et limites des conditions de cet élargissement du champ d'étude que de pointer dans une optique constructive les défaillances que l'on peut constater, les orientations qui devraient être approfondies pour produire du savoir cumulatif, ainsi que d'objectiver de nouveaux sujets pour renouveler la production existante.

Cette entreprise n'est pas gratuite. Sa légitimité me semble résider en effet dans la confluence de plusieurs ordres de facteurs qui militent tous dans le sens de cette mise au point. Au moment où l'on perçoit plus ou moins consciemment un phénomène général de mutation dans l'économie des normativités, on assiste conjointement à une crise des paradigmes d'étude au travers de laquelle aucune discipline ne peut plus désormais à elle seule revendiquer la légitimité d'un discours monopolistique sur les thèmes qui prétendent la circonscrire.

Ce qui explique d'abord qu'on ait vu une série de tentatives de plus en plus explicites de dialogues entre elles pour peu qu'un objet commun les concernât.

Les exemples abondent ces dix dernières années et, s'ils sont loin dans l'ensemble d'avoir été à la hauteur de leurs ambitions ${ }^{1}$, ils ont au moins aidé à promouvoir les conditions d'une conceptualisation efficace de l'interdisciplinarité ${ }^{2}$.

On observera cependant que plus on s'approche de la période actuelle, plus semble se pratiquer avec succès une humble structuration de dialogues, un apprentissage intelligent du dénouement des conditions de la mutuelle surdité des disciplines. Au point que certaines d'entre elles, sans rien renier de qui fonde leur propre spécificité, puisent un regain de vigueur dans la confrontation, avec l'espoir très volontariste de maximiser la rigueur de leurs apports théoriques.

* C.N.R.S./C.E.S.D.I.P., Paris. 
Participe du reste de manière quasi emblématique à cette orientation, la présente revue dont le propos d'étape de $1983^{3}$ rappelait le souci d'oeuvrer à l'unité de la science "tant dans son versant synchronique (sociologique) que diachronique (historique), sans isoler l'un ou l'autre, sous peine de stérilité »; il plaidait pour "une recomposition interdisciplinaire, vue comme un croisement de discours que ces disciplines produisent".

C'est un peu dans cette optique enfin qu'eut lieu les 1er et 2 mars 1984 au C.N.R.S., le premier débat public d'envergure, lié à l'ensemble de cette problématique, à l'occasion du colloque intitulé : "Normes, différences et résistances, régulations et contrôles » qui réunit quarante chercheurs d'horizons différents. Nous en avions avec F. Tétard assurć la synthèse officielle ${ }^{4}$, laquelle obéissait entre autres largement à des considérations de politique scientifique et de logistique.

$\mathrm{Ce}$ que je propose donc ici n'est pas une nouvelle synthèse de ce colloque, mais serait plutôt, en m'inspirant largement des contributions tenues et du matériau recueilli à cette occasion, d'en effectuer une sorte «d'évaluation" qui n'engagerait que son auteur.

La caractéristique essentielle de ce colloque me paraît devoir résider dans les diagnostics différenciés émis (et par suite discutés au fond) sur le sens à donner au constat général "d'une mutation historique de normativités» dans la société française et dont on ne commencerait qu'à peine à sentir les effets.

Je vais tout d'abord essayer d'évaluer les différentes lectures possibles qu'on peut en faire (I) à la lumière de quoi pourra être mieux mesurée par le lecteur lui-même la partie constructive dédiée aux enrichissements attendus de l'interdisciplinarité dans le champ d'étude (II), à comprendre comme relèvement possible au défi lancé aux chercheurs par cette mutation.

\section{Préliminaire d'évaluation}

Mutation historique de normativités, avons-nous dit. Il serait plus juste de l'entendre au pluriel. Elles entretiennent un rapport aux valeurs; elles peuvent également s'appréhender au travers de leur mode de gestion et dans la gamme des sanctions qu'on met en oeuvre.

Et d'abord, d'où viennent-elles, ces mutations ? Est-il possible d'en découvrir le point d'émergence ?

Si l'exercice de datation paraît encore assez périlleux, car l'entreprise risque peut-être d'être trop prématurée, l'accord explicite qui se dégage est celui de rompre de prime abord définitivement avec la liaison courante qui associe le phénomène avec les débuts de la crise économique actuelle ${ }^{5}$. Une grande mutation aurait pu avoir eu lieu beaucoup plus tôt, à savoir vers la fin des années 1950 - début des années 1960. Bon nombre de séries statistiques semblent montrer par exemple, en effet, à cet époque un changement de grandeur dans la gestion des contentieux. C'est également à la fin des années cinquante, qu'on observerait un changement apparemment historique d'une multiplicité d'indicateurs statistiques éloignés les uns des autres, 
ce qui autoriserait à donner un surcroît de consistance au soupçon d'une mutation qualitative sur cette période décisive.

De la même manière, on pourrait trouver des prémisses antérieures à la crise économique de 1974, date symbole qui n'aurait révélé que la réactivation d'une crispation latente sur les situations acquises (on verra par exemple le rôle idéologique considérable de l'attachement à la propriété privée des Français, remontant bien en-deça de cette date).

On a également fait état de ce qu'a pu induire le passage à l'âge adulte en période d'expansion économique. S'il y a toujours eu un problème de la jeunesse depuis que cette catégorie sociale a conquis sa visibilité sociale, on est maintenant à peu près d'accord pour situer une nouvelle formation de son statut et la prise en compte par le corps social de ses valeurs dans la même période fin 50/début 60 .

On doit cependant rester conscient que seule une mise en perspective précise, et non impressionniste, comme on vient de la peindre à gros traits, accompagnée de multichronologies, permettrait d'évaluer le bien fondé de l'hypothèse de ces mutations des registres de normativités. Quoi qu'il en soit, finement resituées et parce qu'elles mettent à jour de longues périodes de stabilité historique, elles peuvent puissamment contribuer - et ce serait déjà un remarquable apport - à détruire l'illusion propre à toute pensée gestionnaire, qui consiste pour «agir», à user de tout temps, d'un discours en forme de "montée quantitative de...».

Outillé par l'ensemble de ces constats non remis en cause dans leur généralité, et puisqu'on raisonne d'abord et surtout à partir de l'actuel, il n'en reste pas moins que la recherche se trouve confrontée au besoin d'expliquer la crise de société qui sous-tend (la) les rupture(s) de normativité(s) considérée(s). Et personne n'ose plus vraiment soutenir aujourd'hui qu'elle serait simplement de l'ordre de la conjoncture.

Si elle est structurelle, comment peut-on au mieux en comprendre le mécanisme ? Autant le dire tout de suite : un diagnostic unanimiste n'a pas pu être fait. Mais au fond, la maladie semble tellement nouvelle que chacun, muni de sa propre spécialité n'a pu que proposer son pronostic. Ce qui n'autorise en rien à le déplorer outre mesure. Car cela pourrait jouer au contraire comme une stimulation majeure à une volonté de s'atteler à la tâche de compréhension, et avec le temps, permettre peut-être un jour de trancher. Mais il est encore bien trop tôt. Voici alors les pronostics qui peuvent se résumer à trois principaux types d'analyse, d'inégale ambition explicative ; deux d'entre eux partent explicitement de l'insécurité ${ }^{6}$, comme principal point de focalisation des interrogations sur les ruptures de normativités...

a) Ou bien, on fait porter l'accent principal sur la mutation générale des valeurs et des conduites dans le corps social; comme elles ne vont plus de soi, ses effets se font ressentir dans les idéologies, lesquelles se trouvent médiatisées par des programmes différenciés de politiques criminelles, selon que les "dirigeants» sont plus ou moins sensibles à certaines plus qu'à d'autres. 
b) Ou bien, on parle de phénomène de "crise générale» et de nouvelle «adaptation des normes». Réduite au phénomène de l'insécurité, cette vision s'expose cependant à la critique d'un fonctionnalisme classique, lequel présuppose un pari optimiste (et conservateur) selon lequel c'est l'adaptation qui résoud la crise ; à la suite d'une période de paroxysme passager (tension forte), c'est le retour à l'état de stabilité homéostatique qui existait antérieurement.

Les sociologues et politologues sont particulièrement réticents à l'emploi de cette dyade crise/adaptation, et préfèrent raisonner en terme de «ruptures et recompositions dans la gestion des normativités », ce qui offre, prétendent-ils, l'avantage de ne pas présager a priori des formes que celles-ci peuvent prendre. Les historiens sont de leur côté plus volontiers réticents à user de concepts qui introduisent les termes de «gestion». Non seulement, cette terminologie ne fait pas partie de leur vocabulaire, car elle leur semble trop connotée par la «séduction des décideurs" '. Mais ils tiennent à préciser, par contre, que le terme de «crise » désigne ici, à leurs yeux, non une crise passagère de "conjoncture", mais bel et bien une véritable "crise de structures». De ce fait, ils ne sont plus tellement éloignés de la conception des sociologues. Ils récusent comme eux, l'idée d'un état d'équilibre idéal fonctionnant par «crise/réadaptation".

C'est pourquoi aussi, la notion de «régulation» devrait être soigneusement réfléchie par les diverses disciplines.

Par-delà ces divergences donc, un consensus s'est finalement dégagé, à partir de cet enjeu d'ordre sociétal qu'il s'agit d'analyser avant tout dans ses aspects les plus généraux (économie, démographie, etc...). Et l'on a paru bien d'accord pour qu'il soit réfléchi sur le long terme, à l'aide d'exemples et d'indicateurs historiques précis. Si un sentiment d'insécurité existe (et doit être analysé), il n'est pas souhaitable d'en faire autre chose qu'un symptôme : l'important, c'est ce qu'il cache.

c) Moins ambitieuse et plus limitée dans ses prétentions enfin - mais pouvant recueillir l'unanimité - la proposition qui, en accord avec le besoin ressenti de mise en historicité des systèmes normatifs, cherche surtout à rendre compte de l'obsolescence et de l'émergence des normes lato sensu, dans une société qui s'accepte en état de crise.

A partir de cette mise au point préliminaire, il devient plus aisé de prendre la mesure des moyens que l'on doit se donner dans le but d'enrichir le champ d'étude.

\section{Les moyens d'enrichir la production scientifique dans le champ d'étude de la déviance et de la criminalité}

Objets inexplorés à combler (1), orientations prioritaires à investiguer de manière plus performante en raison des connaissances déjà disponibles (2), prise en considération de l'apparition de sujets tout à fait novateurs (3), telles paraissent les trois directions essentielles pour remplir cet objectif. 


\section{Les manques}

Les exemples les plus mobilisateurs ont pu être fournis à propos de la (les) police(s), des contentieux et de l'exécution des peines.

\subsection{La police, les polices}

Le constat de carence de la recherche fondamentale interdisciplinaire sur ces «objets» fait incontestablement dans la communauté scientifique l'unanimité ${ }^{8}$. Une évidence s'impose massivement: qu'il s'agisse de recherches sur la police proprement dite, mais aussi sur la gendarmerie, le temps (signe des temps ?) est désormais venu où la situation de carence apparaît intolérable. Quant aux organismes de polices privées et les corps de police municipale, c'est à peine si on a commencé à prendre la mesure de leur existence.

Or, face à la multiplication silencieuse des «polices privées» qui s'est opérée ces dernières années par exemple, il y aurait urgence à commencer par en rendre visible l'existence, en construisant un appareillage statistique sur les sociétés de gardiennage, la géographie de leur implantation et d'étudier la montée historique du phénomène. Il importe avant tout que se développent des recherches empiriques pour cerner les contours de ces "sociétés» privées, parallèlement à ceux du développement intensif des polices municipales dans certaines régions. Enfin, la mise en perspective de ces phénomènes par des comparaisons internationales pourrait sans doute utilement conforter une réflexion plus assurée sur la nature et la forme que prennent les Etats dans le monde occidental.

La situation française apparaît d'autant plus choquante en la matière, que la sociologie de la police, au sens large, se trouve fort en retard vis-à-vis de l'essor remarquable et de considérable productivité offert par ses voisines d'outre Atlantique et européennes (Belgique ou Allemagne par exemple) ${ }^{9}$. Quoi qu'il en soit, une bonne sociologie de la police en France devra nécessairement passer dans les années à venir par l'objectivation de son articulation avec la sécurité privée.

Au plan purement historique, la situation est à peine meilleure. Certes, des travaux sur la police sont en cours chez les historiens sur la première moitié du XIXe et le Second Empire. L'important colloque organisé à Nanterre par Ph. Vigier en décembre 1983 sur le maintien de l'ordre public au XIXe siècle ${ }^{10}$ a montré la voie à suivre. Cependant, il persiste de grandes lacunes tant sur la fin du XIXe que finalement sur tout le XXe siècle. On observe que d'une façon générale les débuts de la IIIe République restent mal élucidés dans les diverses composantes de l'objet. On ne peut donc pas savoir par exemple si la présence au pouvoir des Radicaux a pu offrir historiquement ou non une conception et un usage particuliers de la police qui pourrait suffisamment se différencier d'autres régimes politiques.

Sur un autre plan, on peut observer que les disciplines qui rendent compte des développements de la procédure pénale, dans leur souci de tenir compte des mutations induites par les divers groupes de pression sur son élaboration, gagneraient sans doute beaucoup à focaliser leur attention sur 
le rôle que joue l'institution policière dans son ensemble, aspect là encore passablement négligé jusqu'à présent. On perçoit mieux en effet aujourd'hui à quel point le législateur a toujours plus ou moins été conduit à légaliser des procédures policières auparavant parfaitement illégales.

C'est dire l'importance théorique de cette pratique législatrice qui peut être décrite comme une propension naturelle à aligner le droit sur le fait au nom de l'ineffectivité dénoncée par la police elle-même des règlementations en vigueur. Et par contrecoup détecter le poids essentiel de la police dans les processus de politique criminelle ${ }^{11}$.

A travers cet exemple particulièrement significatif du rôle exemplaire de la police (qu'il serait caricatural de toujours considérer comme un simple instrument dans les mains du pouvoir) se profile la façon dont peuvent se définir peut-être plus généralement les normes juridiques, et par là comment se trouve éclatée la fiction d'un consensus social sur leur élaboration. En somme, que soit définitivement battue en brèche l'illusion idéaliste de la rationalité, censée présider aux destinées du processus juridique normatif.

\subsection{Les contentieux}

Pour continuer dans le sens du constat de carence, s'agissant d'examiner le panorama des sanctions, on est frappé par la diversification croissante et inédite des «techniques» de résolution des conflits, leur éclatement à travers les différentes disciplines du droit existant (qu'il s'agisse du pénal, du civil, de l'administratif) ${ }^{12}$, de la multiplicité vertigineuse des processus de conciliation, qu'ils soient étatiques ou non. La tentation est alors grande de se demander comment la gestion de ces délicats problèmes de contentieux pourra à l'avenir être assurée d'une cohérence de fond, référée par exemple au respect des grands principes libéraux (quid de l'applicabilité d'une règle comme ne bis in idem ?), en même temps que d'une cohérence de procédure, dans les choix civils et politiques (quid du respect des normes internationales secrétées par la Convention Européenne des Droits de l'Homme ou par le pacte des Nations Unies par exemple ?). Questions d'ordre juridique indéniablement problématiques pour qui reste soucieux de raisonner au travers de l'Etat de droit. Si l'interrogation a sa légitimité intrinsèque, la mission du chercheur sera avant tout de prendre l'exacte mesure de ces nouvelles «techniques» de régulation des conflits encore trop imparfaitement analysées du point de vue, sinon de leur réalité, du moins de leurs conséquences sociales ou normatives.

\subsection{L'exécution des peines}

Le domaine de l'exécution des peines, lui aussi reste en grande partie à investiguer :

- Prenons l'objet «emprisonnement». Malgré l'importance des travaux historiques sur la question (XIXe siècle), malgré la pluralité de lectures dont il a déjà fait l'objet (juridique, philosophique, socio-historique) qui font accroire à une bonne connaissance de cette peine, de son institutionnalisation et de ses implications sociales, bien d'autres angles 
d'approches demeurent inexplorés. Par exemple, ces différentes lectures (y compris celle des économistes qui devrait être essentielle) sont loin d'avoir couvert toute la période de son existence. De fait, on ne sait pas grand chose de la prison à l'époque contemporaine et notamment des périodes couvrant la IIIle et IVe République.

- Mais dès lors qu'on aborde dans le même état d'esprit, le contentieux des amendes, l'autre "peine" par excellence, la connaissance de cet objet dans les mêmes composantes que celles ci-dessus décrites, fait figure de nain à côté de l'emprisonnement, promu pour l'occasion, nouveau géant dans la connaissance. Il faut bien constater qu'aucune discipline ne s'est véritablement penchée en profondeur sur ce type de sanction tout aussi fondamentale. Serait-ce à dire alors que son objectivation scientifique ne soit pas «nécessaire» (utile immédiatement) au motif qu'elle n'offrirait pas suffisamment de visibilité sociale problématique?

Mais ce serait alors laisser croire que la recherche sur les processus d'exécution des peines (et on ne vise là que les sanctions judiciaires) ne fonctionnerait que sur des objets aux enjeux directement "politiques ou sociaux » ou du moins qui poseraient symboliquement problème à la conscience collective?

Si ce n'est pas le cas, pour une science sociale qui prétendrait viser plus haut que les contingences immédiates, cette terra incognita reste alors entièrement à défricher.

\section{Des orientations prioritaires pour ajouter aux connaissances disponibles}

On peut les regrouper en trois points successifs encore que l'exercice tenté de regroupement en rubriques soit assez périlleux. Il prend en effet le risque de découper arbitrairement des sujets touchant par nature plusieurs registres à la fois. C'est dans ce domaine là que les controverses furent les plus vives.

\subsection{L'insécurité : point de focalisation général et intérêt scientifique}

Soit, d'abord, le thème de l'insécurité, concept symbole de ralliement commun qui offre la particularité de se diffracter tous azimuts, dès qu'on tente d'en cerner les fondements ${ }^{13}$.

On privilégiera ici les approches les plus globales, celles qui autorisent la description au plan macrosociologique des «mutations» à l'oeuvre dans la société d'aujourd'hui.

La thématique de l'insécurité pose en effet d'emblée le problème de sa cristallisation. Pourquoi, à certains moments un tel sentiment se cristallise$\mathrm{t}$-il dans certaines fractions sociales ? Et d'abord, lesquelles exactement ? S'agit-il par ailleurs d'un phénomène social construit politiquement ? (et si c'est le cas, par qui, pourquoi celui-là, comment ?).

Mais encore, peut-on faire observer, si ce discours sur l'insécurité fonctionne si bien, ne serait-ce pas parce qu'il s'appuie sur des matrices symboliques profondes où sont en jeu des représentations de l'ordre social qui n'iraient plus de soi ? Pourquoi ? Embryon de réponse : Ne s'agirait-il 
pas en fait de l'articulation de l'Etat, de l'ordre social, familial, qui poserait problème aujourd'hui, particulièrement au niveau des représentations dont ces instances font l'objet?

Prenons le même sujet par un autre versant, institutionnel celui-là. Qu'y a-t-il derrière cette cristallisation de l'insécurité, en termes de rapports public/privé ? Comment expliquer au sein de l'Etat français à police publique historiquement forte, la prolifération de «polices privées». Faut-il y voir le signe d'une certaine "désétatisation", alors qu'on observerait à l'inverse depuis une décennie dans les démocraties à Etat traditionnellement faible une "publicisation» de leur police (Grande Bretagne, U.S.A.) ${ }^{14}$ ?

Dans la même perspective, mais sous un aspect plus général, le thème de l'insécurité semble pouvoir être déconstruit et resitué dans le rapport Etat/Société civile. Vu sous cet angle ce rapport aurait l'avantage de subsumer les désirs de recherche interdisciplinaire, puisqu'il autoriserait alors l'examen de deux volets :

- d'un côté, l'état du champ de légitimité ou de délégitimation des différents appareils de prise en charge de la déviance et de la criminalité.

- de l'autre, la manière dont se déploient l'action et les enjeux sociaux effectivement ou symboliquement à l'oeuvre chez les différents acteurs (directement ou non impliqués) de la société civile sur ces questions.

Transition qui nous conduit à aborder une seconde rubrique au sein de laquelle l'accent peut être porté sur la description des systèmes normatifs et des réflexions qu'ils inspirent par rapport aux types de société examinés.

\subsection{Systèmes normatifs et société(s)}

Qui dit «systèmes normatifs" pose immédiatement la question de la pluralité des normes et des valeurs sous-jacentes à ces normes, lesquelles peuvent être d'ordre juridique ou « extra-juridiques ».

Il n'est plus possible, aujourd'hui d'en rester à l'étude de la peine sous prétexte qu'elle délimiterait exclusivement le pénal.

Certes, l'étude des mutations du droit pénal peut être mesurée à l'aune des codifications des valeurs à préserver dans chaque société à un moment donné. Mais on a dit plus haut à quel point il serait hasardeux de rester cantonné à une approche de l'évolution de la sanction pénale sous l'angle du travail linéaire de codification juridique; ou inversement, de se limiter à une approche globale du «système pénal» sans tenir compte de l'évolution des techniques juridiques d'incrimination.

Étudier différentiellement d'un côté les valeurs, de l'autre les sanctions et normes prohibitives serait définitivement s'interdire une réflexion globale sur les valeurs plus générales d'une société et de leur relativisme, sur la part de l'enjeu du pénal par rapport aux autres registres de normes, sur l'impact social de ceux-ci et la dialectique des relations qu'ils entretiennent entre eux.

Et comment rendrait-on compte alors d'un bon nombre de ces interrogations urgentes: quelles sont les normes et valeurs prioritairement véhi- 
culées dans l'Etat, dans le système économique, à tel moment? En quoi sont-elles reflétées ou comment se trouvent-elles ramifiées dans le système de production ou les systèmes familiaux par exemple ? Plus généralement, comment, en amont, les normes sociales deviennent-elles juridiques. Quelle peut être l'adéquation du droit pénal, civil, de la procédure aux pratiques des institutions et à l'évolution des rapports sociaux ? Et en aval, comment les normes juridiques deviennent-elles source de normalisation ? Quelles en sont l'effectivité ou la non-effectivité. Pourquoi ?...

Plus largement, cette série de questions renvoie à la mise en historicité des systèmes normatifs. Pour répondre intelligemment à ces questions, il est impératif de comprendre partout comment les normes en viennent à être constamment «travaillées » par les forces sociales, au point d'émerger et de disparaître, et de résister à d'autres évolutions sociales ?

Toutes ces questions, pas forcément nouvelles, devraient être toujours plus systématisées ou à tout le moins gardées à l'esprit dès lors qu'on est confronté à ce champ. Elles invitent ainsi, tant à écarter l'exclusivisme du terme "pénal» dans la problématique, que d'avoir à répondre à des préoccupations immédiates de prescriptions aux «décideurs».

Car la science sociale doit pouvoir réfléchir sur, et analyser les stratégies des «décideurs" eux-mêmes ${ }^{15}$, en même temps que leur rendre plus visibles certains phénomènes difficiles à percevoir à court terme.

Elle pourrait également trouver des raisons d'être performante et réussir sa mission si, à partir de l'étude des valeurs et des normes, elle parvenait à rendre compte de l'ensemble des phénomènes de transgressions (infractions, déviance...), de sanctions (punitions, réparation, rétribution...), de normalisation,...

A partir de l'ensemble de ces concepts de base qu'elle cherche à utiliser prioritairement, peut s'édifier plus aisément de son côté une connaissance scientifique de la "gestion pénale ${ }^{16}$ qui fasse une plus large part à l'anthropologie.

L'anthropologie (ou l'ethnologie) peuvent en effet puissamment éclairer les notions traditionnelles de transgression et de déviance pour des ensembles définis d'infractions et les différentes sanctions qui s'y rapportent. Les travaux de Verdier et de son équipe ${ }^{17}$ ont bien montré à cet égard la voie à suivre. Et ceci, selon une triple dimension :

- soit, du point de vue du fonctionnement des sociétés sans Etats;

- soit, de celui des sociétés avec Etat, modèles archétypaux pouvant être mis en parallèle sous l'angle des processus généraux de normalisation qui y sont à l'oeuvre :

- mais surtout - et c'est peut-être le plus important - par la mise en évidence de ces processus de normalisation lors de la pénétration d'un modèle normatif dans un autre ${ }^{18}$.

De ce point de vue, peut se développer une étude des effets de l'acculturation autoritaire des droits (pénaux) occidentaux sur des pays comme ceux du Tiers-Monde par exemple ; mais plus généralement, celle des effets 
locaux de la migration d'appareils juridiques, issue des périodes coloniales ou post-coloniales.

Et à l'inverse, demandent à être mis à jour les contre-effets et impacts des phénomènes de migrations de main-d'oeuvre en Europe de l'Ouest, quant à la sécrétion de normes originales internes aussi bien qu'internationales. Comment celles-ci prennent-elles en considération les nouvelles données - valeurs et conduites - du pluralisme culturel produit (dans ses aspects tant conflictuels que régulés) ?

Il serait à cet égard heureux de focaliser l'attention sur les minorités étrangères, comme catégories de populations pouvant aussi bien subir les conflits de cultures que promouvoir en leur sein de nouvelles normes syncrétiques, dont les impacts en "pays d'accueil» seraient à considérer avec attention.

Affirmant cela, on est insensiblement passé par l'effet d'une gradation logique d'un discours sur les systèmes normatifs stricto sensu, à celui des systèmes de valeurs, lesquels feraient réagir les premiers lorsqu'ils sont euxmêmes en proie à de profondes mutations.

Illustration concrète de cette assertion : le phénomène contemporain qu'est l'extension de la catégorie "jeunesse" et son difficile passage à l'état adulte par exemple (passage offrant de multiples configurations) peut tendanciellement provoquer cette mutation des valeurs idéologiques aux conséquences incalculables sur les attitudes de la société tout entière et par suite, sur la logique de l'Etat. Il est dommage que l'énorme investissement qui fut consenti dans les années soixante sur la question se soit un peu essouflé aujourd'hui, comme s'il était des urgences historiques qui déclineraient, passés les phénomènes de mode.

Dès lors, $s^{\prime}$ il apparaît fondé que la recherche fasse porter prioritairement ses efforts sur les instances productrices de normativités - tirant en cela profit des lacunes ou erreurs constatées dans le monde anglo-saxon il reste indispensable qu'elle rende compte du mouvement incessant de leur "transformation" par les nouvelles valeurs véhiculées par les catégories ou agents sociaux des collectivités considérées.

Il convient maintenant de prendre en considération dans un troisième point deux autres voies générales d'étude : l'une qui mettrait plutôt l'accent sur l'économie de la "gestion des normativités», l'autre sur les acteurs vs sujets aux prises avec cette "gestion».

\subsection{Une polarisation sur les "acteurs»}

Face à un processus politique actuel qui cherche à disséminer les pouvoirs de décision, à redistribuer les lieux politiques de responsabilités, selon des critères plus techniques et moins bureaucratiques, des interrogations nouvelles émergent quant à la «gestion» de la diversité des besoins sociaux.

Si une certaine décentralisaiton semble être la réponse politique apportée à la généralisation de situations et de comportements de plus en plus difficilement maîtrisés (les «marges» autrefois circonscrites ne sont plus «marginales»; cf. à cet égard le dérapage des systèmes de protection 
sociale, la prolifération du travail souterrain, etc.) que deviennent les nouveaux dispositifs de «contrôle » de la délinquance par rapport aux nouvelles compétences des instances chargées de les contenir (État central/collectivités locales/réseau associatif) ? Y-a-t-il de nouveaux mécanismes de régulation, de renvoi aux institutions de prise en charge à ces différents niveaux ? Et quelles en seront les interrelations ? Les «sanctions » vont-elles (peuventelles) également entrer dans les faits sous l'habillage de la différence, de la nouveauté, de la non stigmatisation?

Comment se redéploie effectivement la (les) politique(s) criminelle(s) sous cet angle ? Des acteurs sociaux peuvent-ils y participer plus activement en pratique, et comment ?...

Venons-en à une autre manière plus critique de poser ces questions : quelles colorations prennent les différents processus de "disciplinarisation " ${ }^{19}$ dans les domaines qui débordent naturellement le cadre strict de la justice? Par exemple dans le monde de la famille, de l'école, ou celui du travail?

S'il est important d'objectiver les processus globaux de "disciplinarisation» dans la société, il apparaît également utile d'interroger ce qu'est la discipline et cela peut être fait dans l'exemple du monde du travail. Plus précisément, restent inexplorées les perceptions et vécu des règlementations de cette sphère encore fondamentale dans le fonctionnement des sociétés.

Ce serait du reste l'occasion d'un apport substantiel à la réactualisation des recherches générales sur les représentations sociales de la délinquance et des sanctions. Terrain lui-même déjà débroussaillé en sociologie générale, mais qui demanderait cependant à être approfondi au plan diachronique. Car des enquêtes quantitatives réitérées restent les seules à offrir un cadre de référence empirique autorisant principalement, l'interprétation globale des évolutions sociales supposées ${ }^{20}$. Les psycho-sociologues souhaitent quant à eux, apporter leur concours dans cette perspective, pour aider à faire comprendre les niveaux de réalité psychique et/ou symbolique dans les perceptions sur les sanctions; ils peuvent également contribuer à élucider la compréhension des phénomènes de crispation et de repli dans le sentiment d'insécurité actuel. Il reste néanmoins vital, on l'a dit supra - tout en tenant compte de cette dimension - de comprendre et d'expliquer quelles sont les catégories ou couches sociales qui y sont le plus exposées et pourquoi, si l'on ne veut pas retomber dans les errements d'un psychologisme stérilisant.

Ces deux manières d'aborder différenciellement le même thème (sociologiquement/psychologiquement) se retrouvent lorsqu'on examine la question des clientèles des réseaux institutionnels de prise en charge (qu'ils soient répressifs, préventifs, socio-éducatifs, d'aide, d'assistance, etc.).

Pour cerner ce qu'ils "produisent» empiriquement, on cherchera à répondre aux questions suivantes : quels en sont les flux; coment y entret-on et en sort-on ? Quels types de populations y trouve-t-on ? A la suite de quels types d'aiguillage, de quelles filières institutionnelles (sous quelles formes). Selon quelle efficacité ? 
Les réponses données mettront l'accent sur le rôle crucial de l'institution.

Corrélativement, en faisant l'étude des trajectoires individuelles de catégories comme jeunes délinquants, drogués, handicapés ${ }^{21}$, la psychologie serait mieux à même d'expliquer les interactions avec les processus de décision. Ses préoccupations actuelles sont de combiner à la fois l'histoire d'un certain nombre d'individus en rapport avec des contextes de socialisation similaires ou différenciés (histoire événementielle, i.e., relationnelle, sociale) et les diverses sortes de prises en charges sociales. Cela devrait permettre de mieux élucider les processus de prises de décision (qui les prend, quand, selon quels critères ?) leurs aléas (formes, relais et renvois, abandons et surenchères), leurs modalités concrètes d'application (délais, failles, ruptures, décalages, renforcements). Cette approche pourrait également être questionnée sous l'angle des discours idéologiques de légitimation (par exemple, la notion mouvante de «l'intérêt de l'enfant»).

On saluera au passage une sensible évolution caractérisée par la progressive disparition des visions naturalisant les phénomènes de délinquances. On n'ose plus guère aujourd'hui considérer en effet les individus «pris en charge " comme de purs et simples objets de «traitements» et plus généralement comme des objets passifs dans les processus institutionnels de prise en charge. Car, si les institutions ont des rationalités, des fonctions qu'il est nécessaire d'étudier (on s'est beaucoup engagé dans cette voie ces dernières années, par un juste retour de balancier), on doit considérer que les individus (également «acteurs» dans les règles du jeu des «traitements » ou "prises en charges») sont eux aussi des «sous-systèmes ", a-t-on pu dire, porteurs de valeurs et d'intérêts propres; derrière leur subjectivité là aussi, peut se lire la part de leurs besoins.

C'est ce qui explique qu'on puisse parler de "stratégies» (conscientes ou non) derrière l'ensemble des acteurs sociaux, concourrant à, ou luttant contre la reproduction idéologique et matérielle de la réalité sociale.

S'il reste nécessaire d'appréhender les stratégies de chaque acteur, des acteurs dans chaque groupe social, il s'agit en même temps de décrypter les enjeux sociaux globaux du débat sur la gestion des normativités.

Voyons maintenant comment l'interdisciplinarité peut prendre figure sur de tous nouveux objets d'étude.

\section{La promotion de nouveaux sujets pour enrichir le champ}

Faire le point, ce n'est pas seulement définir ou redéfinir des orientations prioritaires de recherche. C'est de surcroît, promouvoir (en les esquissant de façon plus ou moins nuancée) de nouveaux thèmes d'étude capables d'assurer des chances sérieuses de bonne productivité scientifique dans un futur à moyen terme. Nous voudrions rendre hommage à des contributions, d'autant plus remarquables qu'elles proviennent de disciplines ou sous-disciplines jusque là peu impliquées dans la mise en problématique traditionnelle du thème. Elles demandent de ce fait des développements un peu plus larges ${ }^{22}$. Moyennant quoi, elles devraient apporter une substantielle plus- 
value de connaissance scientifique, si elles étaient suivies d'effet, car la pire des attitudes serait certes de ne les considérer que comme de simples exercices de style.

\subsection{Assurance et assurancialisation}

Un historien ${ }^{23}$ avait proposé le thème de «l'assurance » considéré à l'heure actuelle comme faisant partie du "réseau privé de gestion de certains contentieux». Ce sujet entre de plain-pied dans nos préoccupations et offre à l'évidence l'avantage d'être approché sous une très grande diversité de disciplines.

Sur ce thème, que pouvait-on attendre de l'historien ? Son rôle consisterait à montrer que les origines de l'assurance sont liées au concept de catastrophe, plutôt qu'à celui de délit ou de responsabilité dans l'accident. Si l'on part de sa réapparition dans l'Europe Moderne, l'assurance contribue à corriger les aléas de l'exposition aux méfaits qui ne relèvent pas à l'origine du droit (course, piraterie), ou aux pertes fortuites (naufrages, incendies, intempéries...). Elle essaie d'éliminer ou d'éviter les conséquences de la ruine ou de la faillite. Marchands et entrepreneurs sont les premiers à se mettre à l'abri de ces coups du sort (XVIIIe siècle occidental).

C'est aussi à cette époque qu'apparaissent les fraudes destinées à provoquer la perte du bien assuré, et les négligences semi-volontaires ayant pour effet d'accroître le risque couvert.

S'agissant d'apprécier les effets possibles de «l'assurancialisation » ${ }^{24}$ il conviendrait en même temps d'examiner les situations antérieures ou simultanées de non-assurance, et poser comme terrain d'investigation certaines hypothèses ou questions:

- en quoi les contrats qui promettent à terme la jouissance de tel ou tel bien, engagent-ils les mises en demeure ou sanctions contre ceux qui ne le conduiraient pas intacts à terme (exemple de contrats concernant le cheptel ou la réalisation de travaux de transports...) ?

- comment s'organisent les précautions conservatoires, s'engagent les réactions contre la perte suspecte ou le vol déclaré ?

- quels sont les correctifs d'exercice à l'égard de ceux ou celles qui assument la gestion d'êtres ou de biens particulièrement exposés aux dangers (ex. : nourrices, pâtres, gardiens de récoltes, etc...).

D'autre part, une seconde piste d'investigation pourrait être celle de l'étude des contentieux des compagnies d'assurances. Repérée sur le long terme, elle pourrait peut-être révéler des ruptures de solidarité dans les groupements constitués (comme l'entreprise ou la famille); il faudrait s'en assurer en suivant le développement des conditions formulées dans les polices d'assurance et validant le contrat.

Dernière piste enfin : il resterait encore, puisque les assurances adaptent leurs primes à l'accroissement du risque, à résoudre le problème de gestion habituelle pour la société globale, celle-ci devant considérer la tendance aux effets pervers dans la négligence des conduites et l'abolition des 
soucis de sauvegarde; donc, le sacrifice croissant consenti à des garanties individuellement utiles, mais improductives au total. Comparer en outre cette charge aux bénéfices sociaux qui peuvent résulter d'une détente des sollicitudes, celles-ci pouvant au contraire développer des conduites négatives très onéreuses.

De fait, historiens, économistes, sociologues, juristes et anthropologues pourraient-ils, en conservant leurs méthodes et instruments de travail, concourir finalement à expliquer les vagues historiques succesives, à la fois plus ou moins angoissées et plus ou moins généralisées, du besoin de sécurisation sociale, et le phénomène de leurs recompositions pratiques.

\subsection{Sécurité et propriété privée}

Le phénomène idéologique de l'attachement des français à la propriété privée (nourri par la pérennité du Code Civil) est resté ces dernières années, selon un participant politologue considérablement sous-estimée ${ }^{25}$.

Enfermé qu'il était dans les années soixante dans la controverse "embourgeoisement de la classe ouvrière/prolétarisation des classes moyennes", il semblerait que le débat sociologico-politique n'ait pas suffisamment prêté attention à ce phénomène, et ceci permettrait sans doute de mieux expliquer les obstacles que rencontre l'évolution, dans un sens libéral ou permissif, des registres normatifs.

Après avoir examiné les diverses formes de relance de l'aide à la propriété qui parcourent continuellement la Ve République (aussi bien sous les régimes de droite qu'après le changement politique de 1981) il s'agirait maintenant de mesurer les implications de cette diffusion des patrimoines modestes, comme facteur potentiel de conservatisme social. Il faudrait à cet égard vérifier si la norme politique de la C.S.P. (ou P.C.S.) tend à s'imposer chez la plupart des français moins fortement que le système idéologique induit ou associé à la richesse relative de leur foyer. Car il pourrait bien exister une liaison entre stratégie de diversification patrimoniale et expression d'une idéologie méritocratique et conservatrice.

On débouche naturellement sur les systèmes de valeurs dont l'importance a été décrite supra. Ceux-ci risquent de prendre ici un relief d'autant plus exacerbé que la propriété cesse d'être "réservée» et préservée, à l'abri dans des ghettos protégés. Puisqu'elle devient plus largement exposée, elle implique des victimes situées davantage aux échelons inférieurs de la hiérarchie sociale. Ne faudrait-il pas faire alors l'hypothèse que les réactions de ces «victimes» auront des chances d'apparaître d'autant plus violentes que leur idéologie sera plus méritocratique ? ${ }^{26}$.

Là encore, cette piste d'investigation nous paraît très novatrice dans la question qu'elle soulève; qui plus est, elle est prometteuse au plan d'une réponse interdisciplinaire possible, car elle pourrait utilement rassembler des chercheurs politologues, socio-criminologues et historiens du patrimoine par exemple. 


\subsection{Le thème $d u$ « sale boulot» (dirty work)}

Troisième ouverture thématique: le dirty work ${ }^{27}$, thème spécifique issu des pays anglo-saxons (et notamment d'Everett C. Hugues in The Sociological Eye 1971, Men and their work, 1958 ; «Good people and dirty work» in Becker, The other side, 1967...) ${ }^{28}$.

Ce concept de «sale boulot» désigne pour le sens commun l'activité qui consiste à s'occuper des "sales» affaires des autres (qui apparaissent souvent, mais pas toujours à vrai dire comme de "sales gens»).

Il s'agirait dans un premier temps, vue la nouveauté de l'objet inexploré à systématiser, de se poser d'abord des interrogations simples plus que de construire un véritable problématique opérationnelle. La réflexion conduirait à une série de questions non formalisées pouvant être vues comme autant de pistes d'investigations possibles à approfondir par la suite. Nous les restituons telles qu'elles avaient été formulées:

- «considérant qu'on trouve du «sale boulot» dans toutes les professions, quel que soit leur prestige (ex. : personnels de police, gardiens et personnels de la sécurité privée, personnels pénitentiaires, hospitaliers, etc...), on pourrait se demander quelle peut être la part de ce sale boulot par rapport au reste du travail. Comment, en outre, ressort-il des représentations sociales ? ${ }^{29}$.

- le «sale boulot» étant lié aux phénomènes de hiérarchisation interne des professions, quels sont les problèmes de délégation, de fuite par responsabilité, de conflits qu'ils peuvent induire ?

- quel que soit son côté dégradant, le «sale boulot" peut aussi donner du pouvoir. Quel pourrait alors en être la nature?

- ét enfin, quels peuvent être les rapports de ceux qui font le «sale boulot» avec la société globale, ou plus précisément les autres groupes sociaux ?"

Autrement dit, renouer avec la tradition de l'ethnomêthodologie interactionniste pour constituer un matériel de base préalable, serait la première marche à suivre pour déboucher par la suite sur une véritable sociologie des «sales boulots».

En quoi ce thème est-il lui aussi gros de promesses ? Là encore, il ne serait pas trop difficile a priori qu'une réflexion transversale puisse s'instaurer, regroupant un grand nombre de chercheurs de champs différents (lesquels pourraient être des spécialistes de la justice, de la médecine, du travail social et peut-être de l'éducation...).

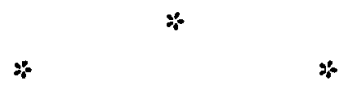

\section{Note d'espoir...}

Au terme de cet itinéraire dont on ne sait si chacun pourra trouver son compte, que dire ? Peut-être simplement ceci : que si ce texte paraît plein de candeur en ce qu'il ne discute pas véritablement des clés de la réussite des pistes qu'il a cherché à promouvoir sur le mode sans doute un peu 
brutal de l'impératif (ou un peu idéaliste du conditionnel), il demeure optimiste, persuadé qu'une interdisciplinarité bien comprise reste la seule solution aux défis lancés à la recherche; et que cet optimisme trouve sa raison d'être à certains signes visibles qui ne peuvent pas tromper désormais; qu'on peut alors se convaincre qu'il ne s'agit plus d'un espoir purement incantatoire.

Frédéric Ocquetean

Centre de recherches sociologiques sur le droit et les institutions pénales

4, rue de Mondovi

F-75C01 Paris

\section{NOTES}

1. Pour une synthèse de ces tentatives de dialogues, voir Ocqueteau et Tétard, 1984, pp. 36-44.

2. Voir Ost et Van de Kerchove, 1982.

3. Déviance et Société, 1983, VII, N 1, pp. 1-2.

4. Ocqueteau, Tétard, 1984, id.

5. Il est clair que la nécessité d'une réflexion sur les chronologies, sur la multiplicité des «périodes contemporaines» (fin XIXe-début XXe siècle) reste à faire.

6. Voir infra II, 2-1.

7. On désigne par « décideurs» aussi bien les instances chargées d'élaborer les politiques criminelles et celles chargées de les mettre en pratique, que tout agent qui, à une place quelconque, "gère" un système de normes quel qu'il soit. On pourrait peut-être y préférer le terme de "gestionnaires» au sens large.

8. Précisons ici qu'en dépit des contributions intéressantes apportées par les chercheurs européens au colloque, nous n'examinons dans cet article que le cas particulier de la France.

9. Voy. les recensions critiques de Van Outrive, Fijnault et De Laet, 1978 et 1984.

10. Travaux en cours de publication pour 1986. A signaler qu'un colloque similaire en mars 1986 prévu par les mêmes organisateurs à Paris, aura pour thème les " victimes des répressions politiques en France et en Europe au XIXe siècle».

11. Voy. Lévy, 1984.

12. Voy. Barberger, 1985.

13. Il convient à cet égard de bien mesurer le fait que le thème n'est devenu un objet d'étude dans les sciences sociales que depuis très récemment en France. Or, non seulement il se manifeste actuellement par la très large ignorance des apports de la littérature scientifique internationale (donc un très grand retard en la matière), mais en plus, il pose deux problèmes majeurs :

- Ou bien il est tellement peu problématisé qu'on évite difficilement le piège des lieux communs;

- ou bien alors, le thème apparaît dans quelques travaux plus sérieux, mais qui se réduisent trop souvent à une sorte de philosophie sociale implicite, qui s'avère très décevante - pour ne pas dire régressive - sur le plan explicatif. 
14. Conceptualisations de l'État puisées chez Badie, Birnbaum (1979, 1982, 1984).

15. Cf. supra note 7.

16. On parle aussi de science de la «politique criminelle», mais le terme n'est pas très heureux; cette "discipline" (ou plutôt ce discours au départ exclusivement juridique) essaie aujourd'hui avec raison de prendre ses distances par rapport au surcodage juridique que son objet d'étude induit. Un essai de conceptualisation a été tenté par Delmas-Marty, 1982.

17. Plus précisément, "le système vindicatoire, esquisse théorique ", pp. 11-42 in Verdier, 1980, et pour un panorama complet, cf. Verdier, 1980; Courtois, 1984 ; et Verdier/Poly, 1984.

18. A signaler entre autres sur ce thème la réunion du 13 janvier 1984 qui s'est tenue à la Maison des sciences de l'homme (Paris), sous l'égide de l'I.A.H.C.C.J. (International Association on the History of Crime and Criminal Justice) et particulièrement la contribution de $P$. Lascoumes sur les Codes napoléoniens en Rhénanie (1814-1848). Voy. plus généralement Zander, Lascoumes, 1984.

19. Qui parle de processus de «disciplinarisation» actuels dit aussitôt : quels sont les «foyers de contestation" possibles dans les groupes d'encadrement de base (exemple : organisations professionnelles comme médecins, personnels de santé, thérapeutes divers, policiers, etc.). S'ils sont des forces dont il conviendrait de mieux cerner les caractéristiques, quelle part jouent-ils dans le développement de ces processus, par exemple.

Poser ainsi ce type de question participe alors d'un exemple supplémentaire de la manière dialectique et non plus linéaire de lire les institutions.

20. En ce sens, cf. les travaux de F. Ocqueteau et $\mathrm{Cl}$. Perez-Diaz sur l'évolution des attitudes de la population française au regard de l'image de la justice, des attitudes de punitivité et des seuils de déviance (recherche en cours au C.E.S.D.I.P.).

21. A signaler pour l'occasion, l'intérêt toujours marqué sur le thème des «populations à hauts risques» (de prise en charges diverses), et la nécessité de recherches comparatives les concernant (on a pu évoquer les immigrés maghrébins). Une discussion s'est élevée quant à la manière de caractériser ces populations. Tout dépend dès lors, d'où l'on se place. Quel est le meilleur critère pour définir le risque ? Et partant de là, quelles populations ? Exemple : si on considère la crise économique, s'agira-t-il des populations les plus exposées à terme, à considérer comme telles (vieillards, propriétaires) ?, ou les maghrébins qui peuvent dès lors être regardés comme constituant un problème plus conjoncturel ?

22. Elles jouent comme un appel d'oxygène, en ce qu'elles renouvellent le champ, et parce qu'elles offrent l'insigne avantage d'avoir été réfléchies dans un esprit d'ouverture interdisciplinaire.

23. Nous reprenons ici les suggestions très structurées d'Yves Castan.

24. Il resterait à bien connaître ses formes de développement empirique à travers l'histoire.

25. On reconnaîtra là, la fameuse thèse de J. Capdevielle (1985, à paraître en 1986) dont les fruits apparaissent prometteurs au plan de la sociologie politique (Capdevielle et al., 1981, Michelat et Simon, 1985). On commence par ailleurs à les tester également au plan de la sociologie des représentations sociales sur la justice (Ocqueteau, Perez-Diaz, note 20). 
26. Cf. en ce sens, les enquêtes de victimisation de Zauberman et Robert (1984, 1986).

27. Proposé par Claudine Herzlich.

28. Sur l'évocation de cet auteur peu connu en Europe de l'Ouest, cf. le récent article de Chapoulie (1984), et pour une vision plus globale du renouveau des enquêtes qualitatives, voy. Poupart, Rains, Pires (1983).

29. En guise d'exemple, on pourrait se demander comment les policiers, le grand public et les dirigeants de la profession perçoivent les fonctions du gardiennage privé ? (sur ces exemples, cf. Shearing/Stenning/Adario, 1985) ; également comment les surveillants de prison se voient-ils entre eux, sont-ils vus par la société, voient-ils les prisonniers et sont-ils vus par eux (sur cet exemple, Casadamont, 1984), etc...

\section{RÉFÉRENCES}

BADIE, B., BIRNBAUM, P., Sociologie de l'État, Paris, Grasset, 1979.

BARBERGER, C., "Justice pénale et administration: le droit de la discipline des codes administratifs ", Année sociologique, 1985, XXXV, pp. 167-178.

BIRNBAUM, P., La logique de l'État, Paris, Fayard, 1982.

BIRNBAUM, P., Dimensions du pouvoir, Paris, P.U.F., 1984.

CAPDEVIELLE, J. et al., France de gauche vote à droite, Paris, Presses de la Fondation Nationale des Sciences Politiques, 1981.

CAPDEVIELLE, J., Le fétichisme du patrimoine; essai sur un fondement de la classe moyenne française, Paris, IEP, 1985.

CASADAMONT, G., La détention et ses surveillants, Paris, thèse E.H.E.S.S., 1984.

CHAPOULIE, J.M., «Everett C. Hugues et le développement du travail de terrain en sociologie ", Revue française de sociologie, 1984, XXV, 4, pp. 582-608.

COURTOIS et al., La vengeance; la vengeance dans la pensée occidentale, Paris, Cujas, 1984.

DELMAS MARTY, M., Modeles et mouvements de politique criminelle, Paris, Economica, 1982.

DÉVIANCE ET SOCIÉTÉ, Propos d'étape, 1983, VII, 1, pp. 1-2.

LÉVY, R., Pratiques policières et processus pénal : le flagrant délit, Paris, C.E.S.D.I.P., 1984

MICHELAT, G., SIMON, M., «Déterminations socio-économiques, organisations symboliques et comportement électoral ", Revue française de sociologie, 1985, XXVI, pp. 32-69.

OCQUETEAU, F., TÉTARD, F., Rapport de la Table ronde CNRS, 1-2 mars 1984, Paris, 1984, "Normes, différences et résistances, régulations et contrôles », ronéo.

OCQUETEAU, F., PEREZ-DIAZ, Cl., L'évolution des attitudes des français sur la justice pénale, recherche en cours, C.E.S.D.I.P.

OST, F., VAN DE KERCHOVE, M., "Jalons pour une épistémologie de la recherche interdisciplinaire en droit", Revue interdisciplinaire d'études juridiques, 1982, 8, PP. 1-8.

POUPART, J., RAINS, P., PIRES, A.P., «Les méthodes qualitatives et la sociologie américaine », Déviance et Société, 1983, VII, 1, pp. 63-91. 
ROBERT, Ph., ZAUBERMAN, R., "Les victimes, la délinquance et l'État, Revue de l'Institut de sociologie, Bruxelles, s.p.

SHEARING, C.D., STENNING, P., ADARIO, S. :

- "Police perceptions of private security", Joumal du Collège canadien de police, 1985, vol. 9, $\mathrm{N}^{\circ} 2$.

- Public perceptions of private security, 1985, ronéo.

- Corporate perceptions of private security, 1985, Center of Criminology, University of Toronto, ronéo.

VAN OUTRIVE, L., FIJNAULT, C., «Recherches sur la police», Déviance et Société, 1978, II, 2, pp. 215-231.

VAN OUTRIVE, L., DE LAET, C., «Recherches sur la police 1978-1982», Déviance et Société, 1984, VIII, 4, Pp. 377-414.

VERDIER, $R$. et al., La vengeance : la vengeance dans les sociétés extra-occidentales, Paris, Cujas, 1980.

VERDIER, R., POLY, J.P., La vengeance: vengeance, pouvoirs et idéologies dans quelques civilisations de l'Antiquité, Paris, Cujas, 1984.

ZANDER, H., LASCOUMES, P., Marx : du vol de bois à la critique du droit, Paris, P.U.F., 1984.

ZAUBERMAN, R., ROBERT, Ph., «Être victime... et après ?», in Le pénal en première ligne ou en dernier ressort, Paris, C.E.S.D.I.P., 1984, ronéo. 